\title{
Nocardia cyriacigeorgica and Aspergillus Co-infection in a Patient with Giant-cell Arteritis
}

\author{
Felisbela Gomes, Pedro La Feria, Catarina Costa, Helena Texeira \\ Unidade Funcional de Medicina 2.1, Hospital de Santo António dos Capuchos, Centro Hospitalar de Lisboa Central, Lisbon, Portugal
}

Received: $16 / 11 / 2018$

Accepted: 06/12/2018

Published: 03/01/2019

\begin{abstract}
How to cite this article: Gomes F, La Feria P, Costa C, Texeira H. Nocardia cyriacigeorgica and aspergillus co-infection in a patient with giant-cell arteritis.
\end{abstract} EJCRIM 2019;6: doi:10.12890/2019_000997.

Conflicts of Interests: The Authors declare that there are no competing interests.

This article is licensed under a Commons Attribution Non-Commercial 4.0 License

\section{ABSTRACT}

Immunosuppressed patients are at greater risk of unusual infections. The authors present the case of a woman with giant-cell arteritis, on oral steroids, who developed cavitating pneumonia due to co-infection with Aspergillus and Nocardia. Reports of such co-infection are rare in the literature. This case highlights the importance of considering rare pathogens in immunosuppressed patients who present with nonspecific symptoms, as well as the impact of such pathogens on clinical management. Another important issue is the need for prophylaxis against Nocardia spp. in immunocompromised patients.

\section{LEARNING POINTS}

- In patients with vasculitis on systemic corticosteroid therapy or other immunosuppressive treatment, suspicion of uncommon infection should increase in parallel with the cumulative dose of these drugs.

- Obtaining an accurate diagnosis and early treatment is essential, but can be very challenging.

- Regular prophylactic therapy should be considered. However, more research is needed to determine whether higher doses of TMP/SMX would provide adequate coverage.

\section{KEYWORDS}

Immunosuppression, Nocardia cyriacigeorgica, Aspergillus

\section{INTRODUCTION}

Immunocompromised patients, particularly those with impaired cell-mediated immunity due to neoplasms, human immunodeficiency virus infection or immunosuppressive therapy, are more susceptible to opportunistic infections ${ }^{[1,2]}$.

Nocardiosis is an uncommon infection caused by Gram-positive aerobic bacteria belonging to the genus Nocardia ${ }^{[3,4]}$. The diagnosis is a challenge because of its non-specific presentation and imaging findings suggesting a broad spectrum of differential diagnoses (Table 1), making microbiological testing important and sometimes difficult ${ }^{[5-8]}$. Recent identification of a species-specific glycoprotein antigen by ELISA allowed detection of antibodies against the antigen in $91 \%$ of cases studied. This molecular technique is the method of choice for speciation $^{[9-13]}$. Mortality rates from pulmonary nocardiosis are about $14-40 \%{ }^{[3,14]}$. Nocardia cyriacigeorgica bacteraemia is responsible for the most serious cases in humans due to both the ability of the organism to infect any organ and also frequent disease progression despite targeted therapy ${ }^{[4]}$. The optimum therapy for nocardiosis is still unclear as no prospective randomized study has determined the most effective therapy. The choice of antimicrobials is based on cumulative experience, investigations in animal models and profiles of in vitro antimicrobial activity ${ }^{[3-4,6,15,16]}$. The incidence of nocardial infections is believed to be increasing worldwide as a result of a growing 
immunocompromised population and improved isolation methods and molecular identification ${ }^{[15-17]}$.

Aspergillosis is the most frequent pulmonary fungal infection. The invasive form is the most severe and can be fatal, thus requiring timely diagnosis and treatment ${ }^{[18,19]}$

Nocardiosis and aspergillosis are typically opportunistic infections but are rarely associated with each other, with few cases described even in the presence of immunosuppression ${ }^{[1,2,9]}$.

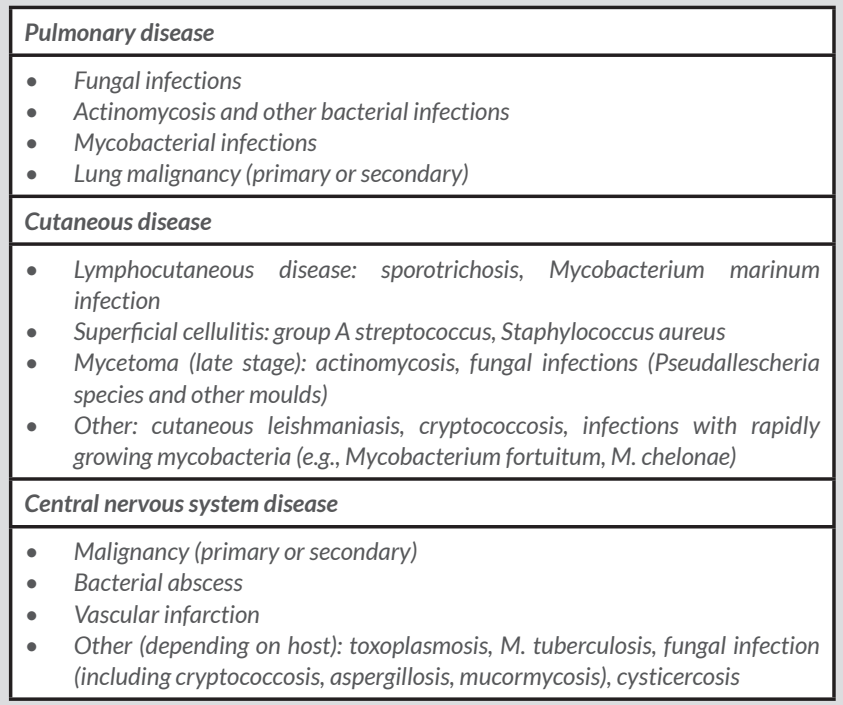

\section{CASE DESCRIPTION}

A 92-year-old woman with type 2 diabetes mellitus, arterial hypertension and dyslipidaemia presented to the hospital. A month previously she had been diagnosed with giant-cell arthritis with left ocular involvement and had started systemic corticosteroid therapy (1 mg/kg/day). She was admitted to hospital with dyspnoea for minor exertion, orthopnoea and a productive cough, which had started 4 days previously. Examination in the emergency room revealed a Glasgow coma scale of 14, hypotension, fever, dehydration and tachypnoea, with an oxygen saturation level of $84 \%$ measured with a pulse oximeter. Blood tests showed a normocytic and normochromic anaemia $(9.0 \times 10 \mathrm{~g} / \mathrm{l})$, without leucocytosis and with relative neutrophilia of 94.5\%, thrombocytopenia of 106,000/l, renal dysfunction (urea $137 \mathrm{mg} / \mathrm{dl}$ and creatinine 1.74 $\mathrm{mg} / \mathrm{dl}$ ), without hepatic enzyme alteration, and a CRP of $406 \mathrm{mg} / \mathrm{l}$. Chest x-ray showed a well-defined shadow in the right lower lung lobe (Fig. 1).

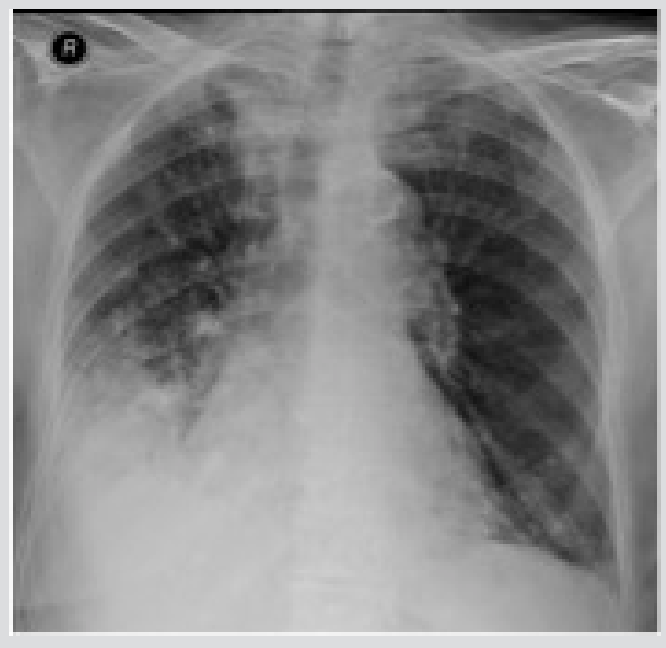


Sepsis was considered to be primarily due to right pneumonia, blood cultures were collected, and double empirical antibiotic therapy with piperacillin-tazobactam and azithromycin was initiated. After 48 hours of antibiotic therapy, the patient's temperature decreased as did the inflammatory parameters, but dependence on oxygen remained and there were signs of respiratory distress.

On the fifth day of hospitalization, the laboratory provided preliminary information concerning possible infection, with actinomyces isolated in blood cultures. The sample was sent to an external laboratory for identification of the microorganism. Thoracic CT revealed multiple foci of pulmonary parenchymal consolidation, present in all segments of the right lung and in the anterior left lobe and basal segments of the left lung, with small areas of cavitation identified within the consolidation areas (Fig. 2). Bronchofibroscopy excluded bronchial tree lesions. On day 11 of the admission, Nocardia cyriacigeorgica was identified in blood cultures and 2 days later Aspergillus was isolated from the bronchoalveolar lavage (BAL) fluid. Tuberculosis, Pneumocystis carinii and HIV were excluded. Therapy was switched to cotrimoxazole and ceftriaxone and, after the results of BAL, voriconazole was added. The fact that there was radiological worsening under piperacillintazobactam therapy supported the hypothesis of pulmonary infection by Nocardia and Aspergillus.

Even with targeted therapy and gradual weaning off steroids, and despite sustained apyrexia and a progressive decrease in inflammatory markers, there was no clinical improvement. On the 25th day of hospitalization, the patient was unresponsive and hypotensive, with worsening dyspnoea and an increasing need for oxygen support. A head CT was performed and excluded any central nervous system complication, while a thoracic CT showed global worsening of the bronchopneumonia as well as cavitated lesions (Fig. 3). Newly collected haemocultures were negative and antibiotherapy was changed to imipenem and linezolid, maintaining co-trimoxazole and voriconazole. Despite the therapeutic optimization, the patient's clinical condition deteriorated with multiple organ failure and she died on the 31st day of hospitalization.

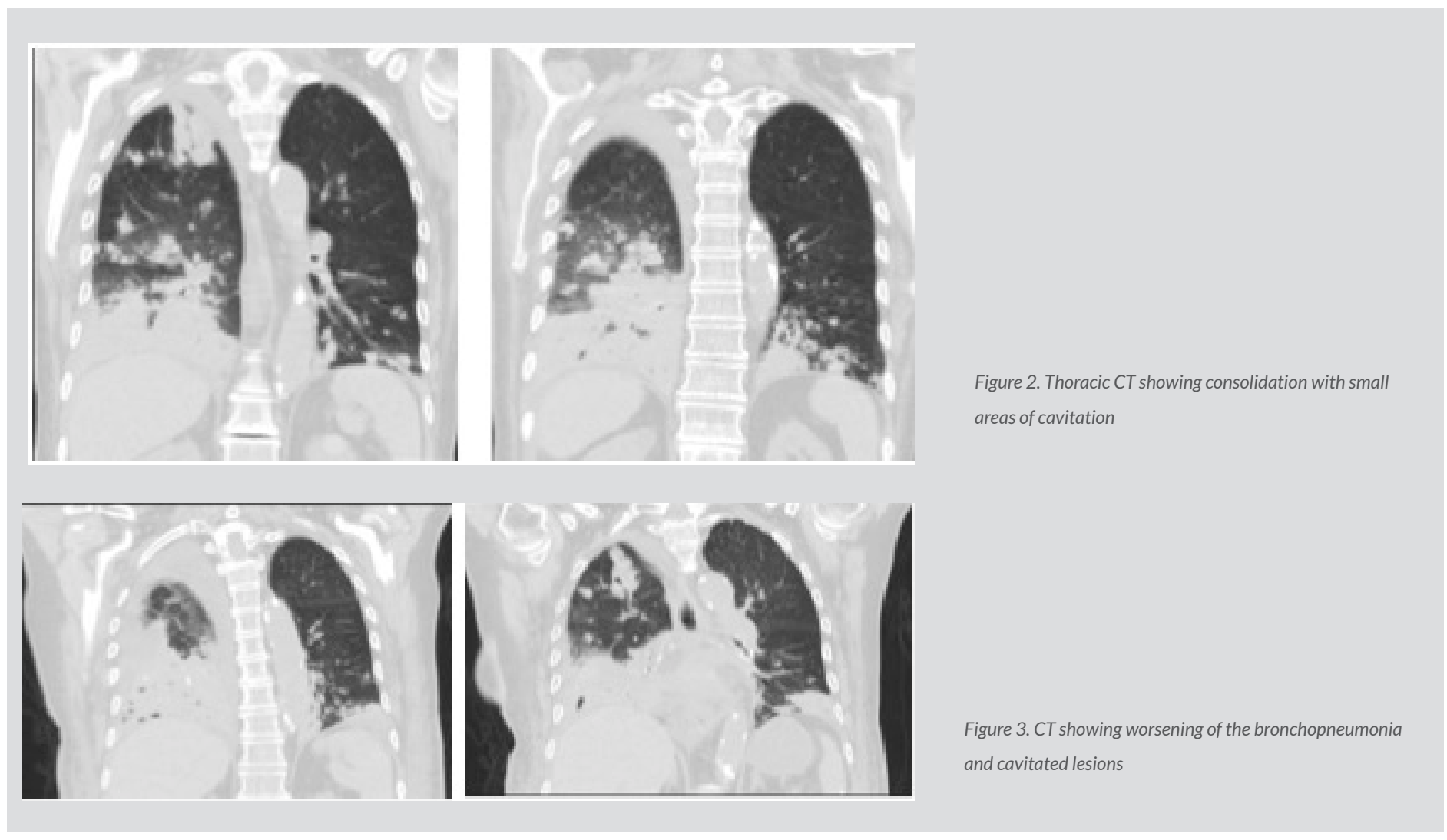

\section{DISCUSSION}

The low specificity of the clinical condition as well as the array of radiological images produced by Nocardia make the diagnosis difficult and the condition is often incorrectly interpreted as pneumonia caused by more common agents (Table 1) ${ }^{[3-8]}$. The diagnosis was difficult because the techniques for identifying the agent were not available in the hospital's laboratory and the blood cultures had to be sent to a specialized centre ${ }^{[10,15-17]}$. 
Identification of Nocardia was only possible on the 11th day of hospitalization and isolation of Aspergillus occurred only on day 13. Both infections are associated with high mortality, even with the early delivery of appropriate therapy. Late initiation of therapy against Nocardia and Aspergillus may have influenced the less favourable outcome of this case. Other factors may have been the late weaning from corticosteroids, considered by some to worsen prognosis, contributing to a less effective response to therapy against both Nocardia and Aspergillus. The fact that the infection did not respond to the combination of ceftriaxone with trimethoprim-sulfamethoxazole, when the isolated strain was sensitive in vitro, suggests a possible discrepancy between in vitro sensitivity and clinical sensitivity as already described in other cases. Clinical improvement is usually observed within 2 weeks after initiation of targeted therapy ${ }^{[19-21]}$. In cases where there is no improvement, combination therapy with a third drug, such as linezolid, can be beneficial[3,4]. Despite therapeutic optimization and exclusion of disseminated infection, thoracic CT showed progression of the infiltrates and the patient died.

This case emphasizes the need to maintain a high index of suspicion, since unusual infections are more likely in this context and usually induce non-specific clinical syndromes. If infection is suspected, empirical therapy should be initiated, even before microbiological results have been received, because early treatment is essential. In this case, empirical coverage should have been considered on day 5 , when blood cultures showed possible actinomyces and thoracic CT showed radiological aggravation under broad-spectrum antibiotic therapy for the most common community-acquired infections. Corticosteroid weaning should have been started early, as this therapy may increase the severity of infection.

The difficulty of making a microbiological diagnosis in routine laboratories should also be emphasized, as well as the consequent problems selecting therapeutic agents and managing immunosuppressive therapy.

The incidence of these infections in patients with systemic vasculitis undergoing immunosuppressive treatment and their prognosis remain unclear. Regular prophylactic therapy should be considered in these patients if their immune status is similar to that of those receiving immunosuppression after organ transplantation ${ }^{[6]}$.

\section{REFERENCES}

1. DP, Parida JR, Chowdhury AC, Agarwal V. Pulmonary co-infection with Nocardia and Aspergillus in a patient with adult-onset Still's disease receiving steroids and tacrolimus. BMJ Case Rep 2014; pii: bcr2014207335, doi: 10.1136/bcr-2014-207335.

2. Hamadani M, Benson DM Jr, Blum W, et al. Pulmonary Nocardia and Aspergillus co-infection in a patient with chronic graft-versus-host disease. Transpl Infect Dis 2008;10:2426.

3. Cooper CJ, Said S, Popp M, Alkhateeb H, Rodriguez C, Porres Aguilar M, Alozie O. A complicated case of an immunocompetent patient with disseminated nocardiosis. Infect Dis Rep 2014;6:5327.

4. Wilson JW. Nocardiosis: updates and clinical overview. Mayo Clin Proc 2012;87:403-407.

5. Yu S, Wang J, Fang Q, Zhang J, Yan F. Specific clinical manifestations of Nocardia: a case report and literature review. Exp Ther Med 2016;12(4):2021-2026.

6. Sirisena D, AI Swedan L, Jayne D, Chakravarty K. A case of systemic nocardiosis in systemic vasculitis and a review of the literature. Singapore Med J 2013;54:e127-e130.

7. Martínez Tomás R, Menéndez Villanueva R, Reyes Calzada S, et al. Pulmonary nocardiosis: risk factors and outcomes. Respirology 2007;12:394.

8. Georghiou PR, Blacklock ZM. Infection with Nocardia species in Queensland. A review of 102 clinical isolates. Med J Aust 1992;156:692.

9. Fatahi-Bafghi M. Nocardiosis from 1888 to 2017. Microb Pathog 2018;114:369-384.

10. van Dam AP, Pruijm MT, Harinck BI, et al. Pneumonia involving Aspergillus and Rhizopus spp. after a near-drowning incident with subsequent Nocardia cyriacigeorgici and N. farcinica coinfection as a late complication. Eur J Clin Microbiol Infect Dis 2005;24:61-64.

11. Roth A, Andrees S, Reiner MK, Harmsen D, Mauch H. Phylogeny of the genus Nocardia based on reassessed $16 \mathrm{~S}$ rRNA gene sequences reveals underspeciation and division of strains classified as Nocardia asteroides into three established species and two unnamed taxons. J Clin Microbiol 2003;41:851-856.

12. Conville PS, Fischer SH, Cartwright CP, Witebsky FG. Identification of Nocardia species by restriction endonuclease analysis of an amplified portion of $16 \mathrm{~S}$ rRNA gene. J Clin Microbiol 2007;45:1146-1151.

13. Namnyak S, Uddin M, Ahmod N. Nocardia cyriacigeorgica bacteraemia presenting with cytomegalovirus disease and rapidly fatal pneumonia in a renal transplant patient: a case report. J Med Case Rep 2011;5:228.

14. Trastoy R, Manso T, García X, Barbeito G, Navarro D, Rascado P, Pérez del Molino ML. Coinfección pulmonar por Nocardia cyriacigeorgica y Aspergillus fumigatus. Rev Esp Quimioter 2017;30:123-126.

15. Salta G, Bouw MJ, Tendeiro T, Saraiva Z, Reis M, Mello e Castro M, Girão A. Nocardiose pulmonar. Acta Médica Portuguesa 2002;15:441-445.

16. Ambrosioni J, Lew D, Garbino J. Nocardiosis: update clinical review and experience at a tertiary center. Infection 2010;38:89-97.

17. Shojaei H, Hashemi A, Heidarieh P, Eshraghi S, Khosravi AR, Daei Naser A. Clinical isolation of Nocardia cyriacigeorgica from patients with various clinical manifestations, the first report from Iran. Med Mycol J 2011;52:39-43.

18. Akashdeep Singh, Deepinder Chhina, Soni RK, Chandan Kakkar, Sidhu US. Clinical spectrum and outcome of pulmonary nocardiosis: 5-year experience. Lung India 2016;33:398403.

19. Peixinho P, Sabino T, Duarte C, Cruz T, Bragança N. Aspergilose pulmonar invasiva. Acta Médica Portuguesa 2003;16:97-99.

20. Valdezate S, Garrido N, Carrasco G, et al. Epidemiology and susceptibility to antimicrobial agents of the main Nocardia species in Spain. J Antimicrob Chemother 2017;72:754.

21. McTaggart LR, Doucet J, Witkowska M, Richardson SE. Antimicrobial susceptibility among clinical Nocardia species identified by multilocus sequence analysis. Antimicrob Agents Chemother 2015;59:269. 